\title{
Optimization of oil palm empty fruit bunches value chain in Peninsular Malaysia
}

\begin{abstract}
Empty fruit bunches (EFB) are valuable palm oil mill waste that could be used to produce multiple products in the form of energy, chemicals, and materials. Therefore, efficient utilization of these biomass resources is essential to optimize the profitability of the industry while addressing environmental issues. In this study, a decision-support tool is developed to perform economic and environmental analyses of the future expansion of the palm oil industry. The sequential steps in the modeling and optimization of the EFB value chain are discussed. This study consists of four processing stages: converting EFB into intermediates and products, transportation networks, direct sale of products, and further processing of products. The proposed tool includes a mathematical model that considers biomass, production, transportation, and emission treatment costs from transportation and production activities. The model is solved with the Advanced Interactive Multidimensional Modeling System to determine the maximum profit and analyze biodiesel production. Peninsular Malaysia is selected as a case study. Results reveal the significant economic benefits of EFB utilization. The most profitable cases of EFB utilization are Case A, C, and D, which have the same $47 \%$ profit margin. The maximum profit of the selected utilization pathways in Case A is USD $151,822,904$ per year based on different ownerships of all EFB processed, which is $79 \%$ lower than the result of a previous study that ignores the capacity limitations of the respective processing facilities. The environment-food-energy-water nexus is also elaborated in this study. The conclusions are obtained based on the limitation, availability, and parameters or data used in this study.
\end{abstract}

Keyword: Optimization; Empty fruit bunch; Biomass value chain; Nexus 